\title{
Hypergeometric Functions and Lucas Numbers
}

\author{
${ }^{1}$ P. Rajkhowa, ${ }^{2}$ Ananta Kumar Bora \\ ${ }^{1,2}$ Department of Mathematics Gauhati University Guwahati-781014,India
}

\begin{abstract}
The main purpose of this paper is to give the relationship between hypergeometric series and the Lucas sequence. A variety of representations in terms of finite sums and infinite series involving coefficients are obtained. While many of them are well known and some identities appear to be now.
\end{abstract}

\section{Hypergeometric Functions:}

The hypergeometric function ${ }_{2} \mathrm{~F}_{1}(\mathrm{a}, \mathrm{b} ; \mathrm{c} ; \mathrm{x})$ is defined by the series

$\sum_{\mathrm{k}=0}^{\infty} \frac{(\mathrm{a})_{\mathrm{k}}(\mathrm{b})_{\mathrm{k}}}{(\mathrm{c})_{\mathrm{k}} \mathrm{k} !} \mathrm{z}^{\mathrm{k}}$

For $|\mathrm{x}|<1$, and by continuous elsewhere (see[2] p.64) where the rising factorial $(\mathrm{a})_{\mathrm{k}}$ is defined by $(\mathrm{a})_{0}=1$ and $(\mathrm{a})_{\mathrm{k}}=\mathrm{a}(\mathrm{a}+1)(\mathrm{a}+2) \ldots . .(\mathrm{a}+\mathrm{k}-1) \quad(\mathrm{k} \geq 1)$

\section{Lucas Numbers}

We define the ${ }^{\text {th }}$ Lucas number, denoted by $\mathrm{L}_{\mathrm{n}}$ as

$$
\mathrm{L}_{\mathrm{n}}=\left(\frac{1+\sqrt{5}}{2}\right)^{\mathrm{n}}+\left(\frac{1-\sqrt{5}}{2}\right)^{\mathrm{n}}
$$

Again we have

$$
{ }_{2} \mathrm{~F}_{1}\left[\begin{array}{c}
\frac{\mathrm{a}}{2}, \frac{\mathrm{a}}{2}+\frac{1}{2} ; \\
\mathrm{z}^{2} \\
\frac{1}{2} ;
\end{array}\right]=\frac{1}{2}\left[(1-\mathrm{z})^{-\mathrm{a}}+(1+\mathrm{z})^{-\mathrm{a}}\right]
$$

Setting $\mathrm{a}=-\mathrm{n}, \mathrm{z}=\sqrt{5}$ we arrive at

$\mathrm{L}_{\mathrm{n}}=\frac{1}{2^{\mathrm{n}-1}}{ }_{2} \mathrm{~F}_{1}\left[\begin{array}{l}-\frac{\mathrm{n}}{2},-\frac{\mathrm{n}}{2}+\frac{1}{2} ; \\ \frac{1}{2} ;\end{array}\right]$

with (1.1)the representation of (2. 3)becomes

$\mathrm{L}_{\mathrm{n}}=\frac{1}{2^{\mathrm{n}-1}} \sum_{\mathrm{k}=0}^{\left\lfloor\frac{\mathrm{n}}{2}\right\rfloor}\left(\begin{array}{c}\mathrm{n} \\ 2 \mathrm{k}\end{array}\right) 5^{\mathrm{k}}$

which is well known ([5].p.168)

Throughout this chapter the following results will be used

$$
\begin{aligned}
& (\alpha)_{2 \mathrm{n}}=2^{2 \mathrm{n}}\left(\frac{\alpha}{2}\right)_{\mathrm{n}}\left(\frac{\alpha+1}{2}\right)_{\mathrm{n}} \\
& (1-\mathrm{n})_{\mathrm{k}}=(-1)^{\mathrm{k}} \frac{(\mathrm{n}-1) !}{(\mathrm{n}-1-\mathrm{k}) !}
\end{aligned}
$$




$$
\begin{aligned}
& (1+\mathrm{n})_{\mathrm{k}}=\frac{(\mathrm{n}+\mathrm{k}) !}{\mathrm{n} !} \\
& \sqrt{\pi} \Gamma(2 \mathrm{z})=2^{2 \mathrm{z}-1} \Gamma(\mathrm{z}) \Gamma\left(\mathrm{z}+\frac{1}{2}\right) \\
& \Gamma(-\mathrm{x})=-\frac{\pi}{\sin \pi \Gamma(\mathrm{x}+1)} \\
& \left(\frac{3}{2}\right)_{k}=\frac{(2 k+1) !}{4^{k} k !}
\end{aligned}
$$

\section{Linear And Quadratic Transformation}

In this section we will use the well-known linear and quadratic transformations for the hypergeometirc functions to derive some representation from (2.3). We begin by the following pair of linear transformations:

$$
\begin{aligned}
& { }_{2} \mathrm{~F}_{1}\left[\begin{array}{ll}
\mathrm{a}, \mathrm{b} ; & \\
\mathrm{c} ; & \mathrm{z}
\end{array}\right]=(1-\mathrm{z})^{-\mathrm{a}}{ }_{2} \mathrm{~F}_{1}\left[\begin{array}{cc}
\mathrm{a}, \mathrm{c}-\mathrm{b} ; \\
& \frac{\mathrm{z}}{\mathrm{z}-1} \\
\mathrm{c} ; &
\end{array}\right] \\
& { }_{2} \mathrm{~F}_{1}\left[\begin{array}{ll}
\mathrm{a}, \mathrm{b} ; \\
\mathrm{z} \\
\mathrm{c} ;
\end{array}\right]=(1-\mathrm{z})^{-\mathrm{b}}{ }_{2} \mathrm{~F}_{1}\left[\begin{array}{l}
\mathrm{a}, \mathrm{c}-\mathrm{a} ; \\
\\
\mathrm{c} ;
\end{array}\right]
\end{aligned}
$$

That are linked together by the relation

$$
{ }_{2} \mathrm{~F}_{1}\left[\begin{array}{ll}
\mathrm{a}, \mathrm{b} ; & \\
\mathrm{c} ; & \mathrm{z}
\end{array}\right]=(1-\mathrm{z})^{\mathrm{c}-\mathrm{a}-\mathrm{b}}{ }_{2} \mathrm{~F}_{1}\left[\begin{array}{c}
\mathrm{c}-\mathrm{a}, \mathrm{c}-\mathrm{b} ; \\
\mathrm{z} \\
\mathrm{c} ;
\end{array}\right]
$$

([6].p.60.Theorem 21)

We also note the obvious relationship

$$
{ }_{2} \mathrm{~F}_{1}\left[\begin{array}{cc}
\mathrm{a}, \mathrm{b} ; \\
\mathrm{c} ;
\end{array}\right]={ }_{2} \mathrm{~F}_{1}\left[\begin{array}{cc}
\mathrm{b}, \mathrm{a} ; & \\
& \mathrm{z} \\
\mathrm{a} ; &
\end{array}\right]
$$

Applying (3.1) to (2.3) then RHS(3.3) of is a finite sum only when $n$ is odd and we get the following identity

$$
\mathbf{L}_{2 n+1}=(-1)^{n}{ }_{2} F_{1}\left[\begin{array}{l}
-n, n+1 ; \\
\frac{5}{2} ;
\end{array}\right]
$$

A companion relationship of (3.4) can be obtained by applying (3.2) to (2.3). In this case $n$ has to be even, and

$$
\mathbf{L}_{2 n}=(-1)^{\mathrm{n}} 2_{2} \mathrm{~F}_{1}\left[\begin{array}{l}
-\mathbf{n}, \mathbf{n} ; \\
\frac{1}{2} ;
\end{array}\right]
$$

Our next transformation formula is 


$$
\begin{aligned}
& { }_{2} \mathrm{~F}_{1}\left[\begin{array}{l}
\mathrm{a}, \mathrm{b} ; \\
\mathrm{z} \\
\mathrm{c} ;
\end{array}\right]=\frac{\Gamma(\mathrm{c}) \Gamma(\mathrm{c}-\mathrm{a}-\mathrm{b})}{\Gamma(\mathrm{c}-\mathrm{a}) \Gamma(\mathrm{c}-\mathrm{b})}{ }_{2} \mathrm{~F}_{1}\left[\begin{array}{l}
\mathrm{a}, \mathrm{b} ; \\
\mathrm{a}+\mathrm{b}-\mathrm{c}+1 ;
\end{array}\right]+(1-\mathrm{z})^{\mathrm{c}-\mathrm{a}-\mathrm{b}} \\
& \frac{\Gamma(\mathrm{c}) \Gamma(\mathrm{a}+\mathrm{b}-\mathrm{c})}{\Gamma(\mathrm{a}) \Gamma(\mathrm{b})}{ }_{2} \mathrm{~F}_{1}\left[\begin{array}{l}
\mathrm{c}-\mathrm{a}, \mathrm{c}-\mathrm{b} ; \\
\mathrm{c}-\mathrm{a}-\mathrm{b}+1 ;
\end{array}\right]
\end{aligned}
$$

However since $a+b-c=-n$ in(2.3), one of the gamma terms in numerator is not defined. So we use the following transformation which is a special case where $\mathrm{a}$ or $\mathrm{b}$ is a negative integer and $\mathrm{m}$ is a non negative integer.

${ }_{2} \mathrm{~F}_{1}\left[\begin{array}{l}\mathrm{a}, \mathrm{b} ; \\ \mathrm{a}+\mathrm{b}+\mathrm{m} ;\end{array}\right]=\frac{\Gamma(\mathrm{m}) \Gamma(\mathrm{a}+\mathrm{b}+\mathrm{m})}{\Gamma(\mathrm{a}+\mathrm{m}) \Gamma(\mathrm{b}+\mathrm{m})}{ }_{2} \mathrm{~F}_{1}\left[\begin{array}{c}\mathrm{a}, \mathrm{b} ; \\ 1-\mathrm{z} \\ 1-\mathrm{m} ;\end{array}\right]$

(see[8].p.559)

Also we use $\sqrt{\pi} \Gamma(2 z)=2^{2 z-1} \Gamma(z) \Gamma\left(z+\frac{1}{2}\right)$

(see[6].p.24)

Then, we have

$$
\begin{aligned}
& { }_{2} \mathrm{~F}_{1}\left[\begin{array}{r}
-\frac{\mathrm{n}}{2}, \frac{\mathrm{n}}{2}+\frac{1}{2} ; \\
5 \\
\frac{1}{2} ;
\end{array}\right]=2^{\mathrm{n}-1}{ }_{2} \mathrm{~F}_{1}\left[\begin{array}{c}
-\frac{\mathrm{n}}{2},-\frac{\mathrm{n}}{2}+\frac{1}{2} ; \\
-4 \\
1-\mathrm{n} ;
\end{array}\right] \\
& \Rightarrow \mathrm{L}_{\mathrm{n}}={ }_{2} \mathrm{~F}_{1}\left[\begin{array}{c}
-\frac{\mathrm{n}}{2},-\frac{\mathrm{n}}{2}+\frac{1}{2} ; \\
-4 \\
1-\mathrm{n} ;
\end{array}\right]
\end{aligned}
$$

Another transformation formula is

$$
\begin{aligned}
& { }_{2} \mathrm{~F}_{1}\left[\begin{array}{l}
\mathrm{a}, \mathrm{b} ; \\
\mathrm{c} ;
\end{array}\right]=\frac{\Gamma(\mathrm{c}) \Gamma(\mathrm{b}-\mathrm{a})}{\Gamma(\mathrm{b}) \Gamma(\mathrm{c}-\mathrm{a})}(-\mathrm{z})^{-\mathrm{a}}{ }_{2} \mathrm{~F}_{1}\left[\begin{array}{l}
\mathrm{a}, 1-\mathrm{c}+\mathrm{a} ; \\
1-\mathrm{b}+\mathrm{a} ;
\end{array}\right]+ \\
& \frac{\Gamma(\mathrm{c}) \Gamma(\mathrm{b}-\mathrm{a})}{\Gamma(\mathrm{a}) \Gamma(\mathrm{c}-\mathrm{b})}(-\mathrm{z})^{-\mathrm{b}}{ }_{2} \mathrm{~F}_{1}\left[\begin{array}{l}
\mathrm{b}, 1-\mathrm{c}+\mathrm{b} ; \\
1-\mathrm{a}+\mathrm{b} ;
\end{array}\right]
\end{aligned}
$$

We apply this to (2.3) and using the fact that $\Gamma(\mathrm{z})$ has poles at non-positive integers, we see that one of two terms in (3.9) always disappears.

Evaluating the gamma terms in the remaining expression we arrive at

$$
\mathrm{L}_{2 \mathrm{n}+1}=(2 \mathrm{n}+1)\left(\frac{5}{4}\right)^{\mathrm{n}}{ }_{2} \mathrm{~F}_{1}\left[\begin{array}{cc}
-\mathrm{n}, \frac{1}{2}-\mathrm{n} ; \\
\frac{3}{2} ;
\end{array}\right]
$$


$\mathbf{L}_{2 \mathrm{n}}=2\left(\frac{5}{4}\right)^{\mathrm{n}}{ }_{2} \mathrm{~F}_{1}\left[\begin{array}{c}-\mathrm{n}, \frac{1}{2}-\mathbf{n} ; \\ \frac{1}{2} ;\end{array}\right]$

(3.11)

From (3.3) we have

$\mathbf{L}_{2 n+1}=(2 n+1)\left(\frac{4}{5}\right)^{n+1}{ }_{2} F_{1}\left[\begin{array}{cr}\frac{3}{2}+\mathbf{n}, 1+n ; \\ \frac{3}{2} ; & \frac{1}{5}\end{array}\right]$

And

$\mathbf{L}_{2 n}=2\left(\frac{4}{5}\right)^{n}{ }_{2} F_{1}\left[\begin{array}{cc}\frac{1}{2}+n, n ; & \frac{1}{5} \\ \frac{1}{2} ; & \end{array}\right]$

(3.13)

These two formulas give us the first infinite series representation for the Lucas Numbers. Employing (3.10) and (3.11) to (3.7) we arrive at

$$
\mathbf{L}_{2 n+1}=5^{n}{ }_{2} F_{1}\left[\begin{array}{cc}
-\mathbf{n}, \frac{1}{2}-\mathbf{n} ; \\
-2 n ; & \frac{4}{5}
\end{array}\right]
$$

$\mathbf{L}_{2 n}=5^{n}{ }_{2} F_{1}\left[\begin{array}{c}-\mathbf{n}, \frac{1}{2}-\mathbf{n} ; \\ 1-2 n ; \frac{4}{5}\end{array}\right]$

(3.15)

The next transformation formula is

$$
\begin{aligned}
& { }_{2} \mathrm{~F}_{1}\left[\begin{array}{c}
\mathrm{a}, \mathrm{b} ; \\
\mathrm{z} \\
\mathrm{c} ;
\end{array}\right]=(1-\mathrm{z})^{-\mathrm{a}} \frac{\Gamma(\mathrm{c}) \Gamma(\mathrm{b}-\mathrm{a})}{\Gamma(\mathrm{b}) \Gamma(\mathrm{c}-\mathrm{a})}{ }_{2} \mathrm{~F}_{1}\left[\begin{array}{c}
\mathrm{a}, \mathrm{c}-\mathrm{b} ; \\
\frac{1}{1-\mathrm{z}} \\
\mathrm{a}-\mathrm{b}+1 ;
\end{array}\right] \\
& +(1-\mathrm{z})^{-\mathrm{b}} \frac{\Gamma(\mathrm{c}) \Gamma(\mathrm{a}-\mathrm{b})}{\Gamma(\mathrm{a}) \Gamma(\mathrm{c}-\mathrm{b})}{ }_{2} \mathrm{~F}_{1}\left[\begin{array}{c}
\mathrm{b}, \mathrm{c}-\mathrm{a} ; \\
\frac{1}{1-\mathrm{z}} \\
\mathrm{b}-\mathrm{a}+1 ;
\end{array}\right]
\end{aligned}
$$

Applying (3.16) to (2.3) we get

$$
\mathbf{L}_{2 n+1}=(2 n+1){ }_{2} F_{1}\left[\begin{array}{cc}
-n, 1+n ; & -\frac{1}{4} \\
\frac{3}{2} ; &
\end{array}\right]
$$

$$
\mathbf{L}_{2 n}=2{ }_{2} F_{1}\left[\begin{array}{rr}
-n, n ; & -\frac{1}{4} \\
\frac{1}{2} ; &
\end{array}\right]
$$

Applying (3.3) to these we get 


$$
\mathbf{L}_{2 n+1}=(2 \mathbf{n}+1) \frac{\sqrt{5}}{2}{ }_{2} F_{1}\left[\begin{array}{rr}
\frac{3}{2}+\mathbf{n}, \frac{1}{2}-\mathbf{n} ; \\
\frac{3}{2} ;
\end{array}\right]
$$

$$
\mathbf{L}_{2 \mathbf{n}}=\sqrt{5}_{2} \mathbf{F}_{1}\left[\begin{array}{c}
\frac{1}{2}+\mathbf{n}, \frac{1}{2}-\mathbf{n} ; \\
\frac{1}{2} ;
\end{array}\right]
$$

Now, we use quadratic transformations.

Our first quadratic transformation is

$$
{ }_{2} \mathrm{~F}_{1}\left[\begin{array}{c}
\mathrm{a}, \mathrm{b} ; \\
\mathrm{z} \\
\mathrm{a}-\mathrm{b}+1 ;
\end{array}\right]=(1+\mathrm{z})^{-\mathrm{a}}{ }_{2} \mathrm{~F}_{1}\left[\begin{array}{c}
\frac{\mathrm{a}}{2}, \frac{\mathrm{a}}{2}+\frac{1}{2} ; \\
\mathrm{a}-\mathrm{b}+1 ;
\end{array}\right.
$$

(see[2].p.128.(3.19))

Employing this to (3.10) and (3.11) we get the following transformations respectively

$$
\mathbf{L}_{2 n+1}=(2 n+1)\left(\frac{3}{2}\right)^{n} \sqrt{\frac{5}{6}}{ }_{2} F_{1}\left[\begin{array}{l}
\frac{1-2 n}{4}, \frac{3-2 n}{4} ; \\
\frac{3}{2} ;
\end{array}\right]
$$

And

$$
\mathbf{L}_{2 \mathbf{n}}=2\left(\frac{3}{2}\right)^{\mathbf{n}}{ }_{2} \mathbf{F}_{1}\left[\begin{array}{cc}
-\frac{\mathbf{n}}{2}, \frac{1-\mathbf{n}}{2} ; & \\
\frac{1}{2} ; & \frac{5}{9}
\end{array}\right]
$$

Again applying (3.3) we get

$$
\mathbf{L}_{2 n+1}=(2 n+1)\left(\frac{2}{3}\right)^{n+1} \sqrt{\frac{5}{6}}{ }_{2} F_{1}\left[\begin{array}{cc}
\frac{5+2 n}{4}, \frac{3+2 n}{4} ; \\
\frac{3}{2} ;
\end{array}\right]
$$

And

$$
L_{2 n}=2\left(\frac{2}{3}\right)^{n} \quad{ }_{2} F_{1}\left[\begin{array}{ccc}
\frac{n+1}{2}, & \frac{n}{2} ; \\
\frac{1}{2} ; & \frac{5}{9}
\end{array}\right]
$$

Employing (3.6) to (3.24) and (3.7) to (3.23) we get

$$
\mathbf{L}_{2 n+1}=(2 n+1) \frac{2^{-\frac{3}{2}}}{3^{n+1}} \sqrt{\frac{5}{6}}{ }_{2} F_{1}\left[\begin{array}{c}
\frac{5+2 n}{4}, \frac{3+2 n}{4} ; \\
\frac{3}{2}+n ;
\end{array}\right]
$$

$$
+\left(\frac{2}{3}\right)^{n+1} 2^{n+\frac{1}{2}} \sqrt{\frac{5}{6}}{ }_{2} F_{1}\left[\begin{array}{c}
\frac{1}{4}-\frac{n}{2},-\frac{1}{4}-\frac{n}{2} ; \\
\frac{1}{2}+n ;
\end{array}\right]
$$


$\mathbf{L}_{2 \mathbf{n}}=3^{\mathrm{n}}{ }_{2} \mathrm{~F}_{1}\left[\begin{array}{r}-\frac{\mathbf{n}}{2}, \frac{1-\mathbf{n}}{2} ; \\ 1-\mathbf{n} ;\end{array}\right]$

From (3.3) and (3.26) we get

$L_{2 n+1}=\frac{2 n+1}{4.3^{n+\frac{1}{2}}}{ }_{2} F_{1}\left[\begin{array}{cc}\frac{1}{4}+\frac{n}{2}, \frac{3}{4}+\frac{n}{2} ; & \\ \frac{3}{2}+n ; & \frac{4}{9}\end{array}\right]$
$+\frac{2^{2 n+\frac{3}{2}}}{3^{5 n+2}} \cdot 5^{2 n+1}{ }_{2} F_{1}\left[\begin{array}{c}\frac{1}{4}+\frac{3 n}{2}, \frac{3}{4}+\frac{3 n}{2} ; \\ \frac{1}{9}+n ;\end{array}\right]$

Again applying(3.1) to (3.26) we get

$\mathbf{L}_{2 n+1}=\frac{3(2 n+1)}{4} 5^{-\frac{3}{4}-\frac{n}{2}}{ }_{2} F_{1}\left[\begin{array}{c}\frac{5+2 n}{4},+\frac{3+2 n}{4} ; \\ \frac{3}{2}+n ;\end{array}\right]$

$+\left(\frac{2}{3}\right)^{2 n+1} \cdot 5^{\frac{1}{4}+n}{ }_{2} F_{1}\left[\begin{array}{c}\frac{1}{4}-\frac{n}{2}, \frac{3}{4}+\frac{3 n}{2} ; \\ \frac{1}{2}+n ;\end{array}\right]$

For odd $n$ we have from (3.27)by using (3.1) that

$$
\mathbf{L}_{4 \mathbf{n}+2}=2\left(\frac{\sqrt{5}}{2}\right)^{2 n+1}(2 \mathbf{n}) !{ }_{2} \mathbf{F}_{1}\left[\begin{array}{rr}
-\mathbf{n}-\frac{1}{2},-\mathbf{n} ; \\
-2 \mathbf{n} ;
\end{array}\right]
$$

Again for even n, employing (3.2) to (3.27) we arrive at

$$
\mathbf{L}_{4 \mathrm{n}}=2 \frac{6}{\sqrt{5}}\left(\frac{5}{4}\right)^{\mathrm{n}}(2 \mathrm{n}-1){ }_{2} \mathrm{~F}_{1}\left[\begin{array}{r}
\frac{1}{2}-\mathrm{n}, 1-\mathrm{n} ; \\
1-2 \mathrm{n} ;
\end{array}\right]
$$

Again the identity (3.29) can be further transformed by formula (3.3) as follows

$$
\begin{aligned}
& \mathbf{L}_{2 n+1}=\frac{2 n+1}{4} \cdot 5^{-\frac{1}{4}-\frac{n}{2}}{ }_{2} F_{1}\left[\begin{array}{l}
\frac{1}{4}+\frac{n}{2}, \frac{3}{4}+\frac{n}{2} ; \\
\frac{3}{2}+n ;
\end{array}\right]+ \\
& \frac{1}{3} \cdot\left(\frac{2}{3}\right)^{2 n+1} 5^{\frac{3}{4}+n}{ }_{2} F_{1}\left[\begin{array}{l}
\frac{1}{4}+\frac{3 n}{2},-\frac{1}{4}-\frac{n}{2} ; \\
\frac{1}{2}+n ;
\end{array}\right]
\end{aligned}
$$

Applying (3.16) to identity (3.23) we get 


$$
\mathbf{L}_{4 n+2}=(-1)^{n} 3(2 n+1){ }_{2} F_{1}\left[\begin{array}{ll}
-n, n+1 ; & \frac{9}{4} \\
\frac{3}{2} ; &
\end{array}\right]
$$

And

$$
\mathbf{L}_{4 n}=2(-1)^{n}{ }_{2} F_{1}\left[\begin{array}{ll}
-n, n ; & \frac{9}{4} \\
\frac{1}{2} ;
\end{array}\right]
$$

Again applying (3.1) to these two identities we arrive at

$$
\begin{aligned}
& L_{4 n+2}=3(2 n+1)\left(\frac{5}{4}\right)^{n}{ }_{2} F_{1}\left[\begin{array}{ll}
-n, \frac{1}{2}-n ; & \\
\frac{3}{2} ; & \frac{9}{5}
\end{array}\right] \\
& L_{4 n}=2\left(\frac{5}{4}\right)^{n}{ }_{2} F_{1}\left[\begin{array}{ll}
-n, \frac{1}{2}-n ; \\
\frac{1}{2} ;
\end{array}\right]
\end{aligned}
$$

Finally we use the following two quadratic transformation formulae

$$
\begin{aligned}
& { }_{2} \mathrm{~F}_{1}\left[\begin{array}{c}
\mathrm{a}, \mathrm{b} ; \\
\mathrm{z} \\
\mathrm{a}-\mathrm{b}+1 ;
\end{array}\right]=(1-\mathrm{z})^{-\mathrm{a}}{ }_{2} \mathrm{~F}_{1}\left[\begin{array}{c}
\frac{\mathrm{a}, \frac{\mathrm{a}-2 \mathrm{~b}+1}{2} ;}{\mathrm{a}-\mathrm{b}+1 ;}-\frac{4 \mathrm{z}}{(1-\mathrm{z})^{2}} \\
]
\end{array}\right] \\
& { }_{2} F_{1}\left[\begin{array}{c}
a, b ; \\
z \\
a-b+1 ;
\end{array}\right]=\frac{1+z}{(1-z)^{a+1}}{ }_{2} F_{1}\left[\begin{array}{c}
\frac{1+a}{2}, \frac{a}{2}-b+1 ; \\
-\frac{4 z}{(1-z)^{2}} \\
a-b+1 ;
\end{array}\right]
\end{aligned}
$$

Applying these two to (2.3) we get

$$
\mathbf{L}_{4 n+2}=3_{2} F_{1}\left[\begin{array}{r}
-\mathbf{n}, \mathbf{n}+1 ; \\
-\frac{5}{4} \\
\frac{1}{2} ;
\end{array}\right]
$$

IV.

\section{Explicit Formulas}

In this section we will simply rewrite the formulas obtained above in terms of combinatorial sums. Identities (3.8), (3.17) and (3.18) respectively lead to the sum

$$
\mathrm{L}_{\mathrm{n}}=\sum_{\mathrm{k}=0}^{\left\lfloor\frac{\mathrm{n}}{2}\right\rfloor} \frac{\mathrm{n}}{\mathrm{n}-\mathrm{k}}\left(\begin{array}{c}
\mathrm{n}-\mathrm{k} \\
\mathrm{k}
\end{array}\right)
$$




$$
\begin{aligned}
& \mathrm{L}_{\mathrm{n}+1}=\sum_{\mathrm{k}=0}^{\mathrm{n}} \frac{2 \mathrm{n}+1}{2 \mathrm{k}+1}\left(\begin{array}{c}
\mathrm{n}+\mathrm{k} \\
2 \mathrm{k}
\end{array}\right) \\
& \mathrm{L}_{2 \mathrm{n}}=2 \sum_{\mathrm{k}=0}^{\mathrm{n}} \frac{\mathrm{n}}{\mathrm{n}+\mathrm{k}}\left(\begin{array}{c}
\mathrm{n}+\mathrm{k} \\
2 \mathrm{k}
\end{array}\right)
\end{aligned}
$$

Again Identities (2.3), (3.40) and (3.5) give rise to

$$
\begin{aligned}
& \mathrm{L}_{\mathrm{n}}=\frac{1}{2^{\mathrm{n}-1}} \sum_{\mathrm{k}=0}^{\frac{\mathrm{n}}{2}}\left(\begin{array}{c}
\mathrm{n} \\
2 \mathrm{k}
\end{array}\right) 5^{\mathrm{k}} \\
& \mathrm{L}_{4 \mathrm{n}}=\sum_{\mathrm{k}=0}^{\mathrm{n}} \frac{2 \mathrm{n}}{\mathrm{n}+\mathrm{k}}\left(\begin{array}{c}
\mathrm{n}+\mathrm{k} \\
2 \mathrm{k}
\end{array}\right) 5^{\mathrm{k}} \\
& \mathrm{L}_{2 \mathrm{n}}=\sum_{\mathrm{k}=0}^{\mathrm{n}}(-1)^{\mathrm{n}+\mathrm{k}} \frac{2 \mathrm{n}}{\mathrm{n}+\mathrm{k}}\left(\begin{array}{c}
\mathrm{n}+\mathrm{k} \\
2 \mathrm{k}
\end{array}\right) 5^{\mathrm{k}}
\end{aligned}
$$

Both (3.4) and (3.14) lead to the following identity

$$
\mathrm{L}_{2 \mathrm{n}+1}=\sum_{\mathrm{k}=0}^{\mathrm{n}}(-1)^{\mathrm{n}+\mathrm{k}}\left(\begin{array}{c}
\mathrm{n}+\mathrm{k} \\
2 \mathrm{k}
\end{array}\right) 5^{\mathrm{k}}
$$

(4.7)

Again both (3.30) and (3.39) lead to the following

$$
\mathrm{L}_{4 \mathrm{n}+2}=3 \sum_{\mathrm{k}=0}^{\mathrm{n}}\left(\begin{array}{c}
\mathrm{n}+\mathrm{k} \\
\mathrm{k}
\end{array}\right) 5^{\mathrm{k}}
$$

Again from (3.23) and (3.27) we get respectively

$$
\begin{aligned}
& \mathrm{L}_{2 \mathrm{n}}=2\left(\frac{3}{2}\right)^{\mathrm{n}} \sum_{\mathrm{k}=0}^{\left.\frac{\mathrm{n}}{2}\right\rfloor}\left(\begin{array}{c}
\mathrm{n} \\
2 \mathrm{k}
\end{array}\right)\left(\frac{5}{9}\right)^{\mathrm{k}} \\
& \mathrm{L}_{2 \mathrm{n}}=3^{\mathrm{n}} \sum_{\mathrm{k}=0}^{\left\lfloor\frac{\mathrm{n}}{2}\right\rfloor}(-1)^{\mathrm{k}} \frac{\mathrm{n}}{\mathrm{n}-\mathrm{k}}\left(\begin{array}{c}
\mathrm{n}-\mathrm{k} \\
\mathrm{k}
\end{array}\right) 9^{\frac{1}{\mathrm{k}}}
\end{aligned}
$$

From the identities (3.19), (3.20),(3.12) and (3.13) we respectively arrive at

$$
\mathrm{L}_{2 \mathrm{n}+1}=\sqrt{5} \sum_{\mathrm{k}=0}^{\infty}\left(\begin{array}{c}
\mathrm{n}+\mathrm{k}+\frac{1}{2} \\
2 \mathrm{k}+1
\end{array}\right)
$$

$$
\mathrm{L}_{2 \mathrm{n}}=\sqrt{5} \sum_{\mathrm{k}=0}^{\infty}\left(\begin{array}{c}
\mathrm{n}+\mathrm{k}-\frac{1}{2} \\
2 \mathrm{k}+1
\end{array}\right)
$$

$$
\mathrm{L}_{2 \mathrm{n}+1}=(2 \mathrm{n}+1)\left(\frac{4}{5}\right)^{\mathrm{n}+1} \sum_{\mathrm{k}=0}^{\infty}\left(\begin{array}{c}
2 \mathrm{n}+2 \mathrm{k}+1 \\
2 \mathrm{k}
\end{array}\right) \frac{1}{2 \mathrm{k}+1}\left(\frac{1}{5}\right)^{\mathrm{k}}
$$


$\mathrm{L}_{2 \mathrm{n}}=2\left(\frac{4}{5}\right)^{\mathrm{n}} \sum_{\mathrm{k}=0}^{\infty}\left(\begin{array}{c}2 \mathrm{n}+2 \mathrm{k}-1 \\ 2 \mathrm{k}\end{array}\right) \frac{1}{5^{\mathrm{k}}}$

(4.14)

Using (3.22),(3.24) and (3.25) respectively we arrive at

$\mathrm{L}_{2 \mathrm{n}+1}=(2 \mathrm{n}+1)\left(\frac{3}{2}\right)^{\mathrm{n}} \sqrt{\frac{5}{6}} \sum_{\mathrm{k}=0}^{\infty}\left(\begin{array}{c}\mathrm{n}-\frac{1}{2} \\ \mathrm{k}\end{array}\right) \frac{1}{2 \mathrm{k}+1}\left(\frac{5}{9}\right)^{\mathrm{k}}$

$\mathrm{L}_{2 \mathrm{n}+1}=(2 \mathrm{n}+1)\left(\frac{2}{3}\right)^{\mathrm{n}+1} \sqrt{\frac{5}{6}} \sum_{\mathrm{k}=0}^{\infty}\left(\begin{array}{c}\mathrm{n}+2 \mathrm{k}+\frac{1}{2} \\ 2 \mathrm{k}\end{array}\right) \frac{1}{2 \mathrm{k}+1}\left(\frac{5}{9}\right)^{\mathrm{k}}$

$\mathrm{L}_{2 \mathrm{n}}=2\left(\frac{2}{3}\right)^{\mathrm{n}} \sum_{\mathrm{k}=0}^{\infty}\left(\begin{array}{c}\mathrm{n}+2 \mathrm{k}-1 \\ 2 \mathrm{k}\end{array}\right)\left(\frac{5}{9}\right)^{\mathrm{k}}$

V. Further Application

The Generalized Hypergeometirc Function is defined by

${ }_{\mathrm{p}} \mathrm{F}_{\mathrm{q}}\left[\begin{array}{l}\alpha_{1}, \alpha_{2} \ldots \ldots, \alpha_{\mathrm{p}} ; \\ \beta_{1}, \beta_{2} \ldots \ldots, \beta_{\mathrm{p}} ;\end{array}\right]=1+\sum_{\mathrm{n}=1}^{\infty} \frac{\prod_{\mathrm{i}=1}^{\mathrm{p}}\left(\alpha_{\mathrm{i}}\right)_{\mathrm{n}}}{\prod_{\mathrm{i}=1}^{\mathrm{q}}\left(\beta_{\mathrm{j}}\right)_{\mathrm{n}}} \frac{\mathrm{z}^{\mathrm{n}}}{\mathrm{n} !}$

(see[6].p.73)

We use the following identity due to Clausent (see[8].p.180)

$$
\left\{{ }_{2} F_{1}\left[\begin{array}{lr}
a, b ; & z \\
a+b+\frac{1}{2} ;
\end{array}\right]\right\}^{2}={ }_{3} F_{2}\left[\begin{array}{r}
2 a, a+b, 2 b ; \\
z \\
a+b+\frac{1}{2}, 2 a+2 b
\end{array}\right]
$$

Taking $\mathrm{a}=-\mathrm{n}, \mathrm{b}=1+\mathrm{n}, \mathrm{Z}=-\frac{1}{4}$ then (3.17) and (5.2) gives

$$
\mathbf{L}_{2 n+1}^{2}=(2 n+1)^{2}{ }_{3} F_{2}\left[\begin{array}{r}
-2 n, 1,2+2 n ; \\
-\frac{1}{4} \\
\frac{3}{2}, 2 ;
\end{array}\right]
$$

By way of (5.1) we get

$$
\mathrm{L}_{2 \mathrm{n}+1}^{2}=(2 \mathrm{n}+1)^{2} \sum_{\mathrm{k}=0}^{2 \mathrm{n}}\left(\begin{array}{c}
2 \mathrm{n}+\mathrm{k}+1 \\
2 \mathrm{k}+1
\end{array}\right) \frac{1}{\mathrm{k}+1}
$$

\section{References}

[1] A.K.Agarwal, “On a new kind of numbers", The Fibonacci Quarterly, Vol 28(3), ( 1990), 194-199

[2] G.E. Andrews, Richard Askey, Ranjan Roy "Special Functions",Cambridge University press 1999.

[3] W.N. Bailey. “ Generalized Hypergeometric Series". Cambrige: Cambridge University Press, 1935.

[4] C.Jordan. "Calculus of Finite Differences". New York : Chelsea, 1950.

[5] Thomas Koshy. "Fibonacci and Lucas Numbers with Applications", 2001.

[6] E.D. Rainville. “'Special Functions", New York : Macmillan, 1967.

[7] J.Riordan. “Cambinatorial Identities". Huntington. New York: Krieger, 1979.

[8] M.Abramowitz and I.A.Stegun. “Handbook of Mathematical Functions". Washington, D.C.: National Bureau of standards, 1964. 